\title{
Using Environmental Models to Optimize Sensor Placement
}

\author{
Rustam Stolkin, Lucas Vickers, and Jeffrey V. Nickerson
}

\begin{abstract}
Sensors used in protective applications are conventionally placed on perimeters or over areas in an evenly distributed pattern. However, such patterns may actually be suboptimal, since environmental factors may make some forms of attack more or less likely than others. We describe a protective application of sensors for detecting underwater threats in an urban estuary environment. We demonstrate that environmental information, derived from a computational river current model, can be utilized to optimize sensor placement, increasing detection rates and decreasing the number of required sensors. Simulation results show a significant improvement in detection likelihood for a given number of sensors; alternatively, fewer sensors can be used while still maintaining the detection rate of a conventional approach.
\end{abstract}

Index Terms-Diver detection, environmental modeling, harbor security, port security, sensor optimization, sensor placement.

\section{INTRODUCTION}

D ETECTING and tracking moving objects is difficult. Sensory data and other information are often sparse or incomplete and are always associated with a degree of uncertainty [1] which may, itself, be hard to estimate.

Sometimes it is possible to incorporate additional information into decision making by combining prior knowledge of the environment in which the object is moving with a statistical model of the object's behavior in response to that environment. In [2], mobility and terrain analysis are used to predict possible movement plans for targets.

An interesting example is the case of detecting and tracking intentionally moving objects in a river, since rivers contain distinctive and varying currents which impose constraints on the objects' motion. Current data can be derived from a computational estuarine model [3].

Our previous work has explored different configurations of both moving, [4], and stationary, [5], sensors. To our knowledge, no previous work considers the effects of current speeds on intentionally moving objects, and in turn how sensor networks should be designed to take advantage of those effects.

\section{Probability of Detection}

We consider the case of detecting a diver who crosses a linear arrangement of sensors stretched across a river. In particular,

Manuscript received May 27, 2006; accepted September 6, 2006. This work was supported by the Office of Naval Research, Swampworks, under Grant N00014-05-1-00632. The associate editor coordinating the review of this paper and approving it for publication was Prof. Ralph Etienne-Cummings.

R. Stolkin is with the Center for Maritime Systems, Stevens Institute of Technology, Hoboken, NJ 07030 USA (e-mail: rstolkin@ stevens.edu).

L. Vickers is with the Department of Computer Science, Stevens Institute of Technology, Hoboken, NJ 07030 USA (e-mail: lvickers@ stevens.edu).

J. V. Nickerson is with the Howe School of Technology Management, Stevens Institute of Technology, Hoboken, NJ 07030 USA (e-mail: jnickerson@ stevens. edu).

Color versions of one or more of the figures in this paper are available online at http://ieeexplore.ieee.org.

Digital Object Identifier 10.1109/JSEN.2006.890148 we consider the case of a diver swimming south in the Hudson river against northbound currents. We denote the conditional probability that the $i^{\text {th }}$ sensor will detect the diver, given that he crosses the line of sensors at a particular point $x$ by $P\left(D^{i} \mid x\right)$. Assuming that the performance of each sensor is independent of the others, it is easy to show that the total probability that a diver, crossing at point $x$, be detected by the entire set of sensors is given by

$$
P\left(D^{T} \mid x\right)=1-P\left(\bar{D}^{T} \mid x\right)=1-\prod_{i}\left\{1-P\left(D^{i} \mid x\right)\right\} .
$$

The terms, $P\left(D^{i} \mid x\right)$, are calculated using a simple sensor model in which probability of detection is approximated by a linear decrease with range from a maximum value of $95 \%$ to reflect the fact that detection is never guaranteed. This model is based on recent work on passive diver detection [6], which suggests that divers can be detected by thresholding a feature value derived from a passive acoustic hydrophone signal. Note that the technique described here can accommodate any detection versus range sensor model. Hence, we can define

$$
P\left(D^{i} \mid x\right)=0.95\left(1-\frac{\left|x-x_{i}\right|}{50}\right), 0 \leq\left|x-x_{i}\right|<50
$$

where $x_{i}$ denotes the position of the sensor and the maximum detection range is $50 \mathrm{~m}$. We now examine the joint probability that a diver chooses to cross the line of sensors at a particular point $x$ and is detected when he does, so

$$
P\left(D^{T}, x\right)=P(x) P\left(D^{T} \mid x\right)=P\left(D^{T} \mid x\right) p(x) \delta x
$$

where $p(x)$ is the probability density function which describes the likelihood that locations $x$ along the sensor line will be the site of an attempted crossing by the diver. The term $p(x)$ is defined by a "diver preference" curve, a simple function of current speed based on advice from our expert divers. We use a log-normal distribution to reflect the fact that it is impossible for the diver to swim against currents beyond $0.5 \mathrm{~ms}^{-1}$ (treated as negative current speeds), and also that the diver will not wish to travel at very high speeds (with the current) in the highly turbid and cluttered river environment. In contrast, a conventional Gaussian distribution is not able to model zero probabilities for large negative current speeds. Hence

$$
p(x)=f\left(v_{x}\right)=\frac{1}{\left(v_{x}+0.5\right) \sigma \sqrt{2 \pi}} e^{-\left\{\ln \left(v_{x}+0.5\right)-\mu\right\}^{2} / 2 \sigma^{2}}
$$

where the river current speed $\left(v_{x}\right)$ at location $x$ is found from the current profile data output by our computational estuarine model [3]. This model forecasts current speeds for locations on a grid of cells, spanning the lower Hudson river and New York harbor. We generate the current curve $v_{x}=f(x)$ by least-squares fitting a polynomial to current values forecast for a line of cells across the river (Fig. 1). We can now evaluate the effectiveness 


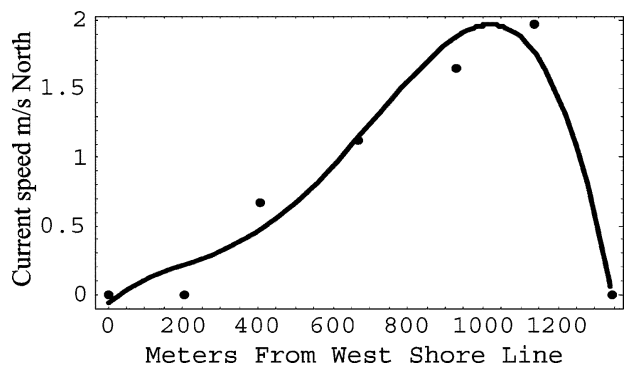

Fig. 1. Current speeds for cross section of Hudson river. Polynomial interpolation of forecast current speeds from the NYHOPS computational estuarine model.

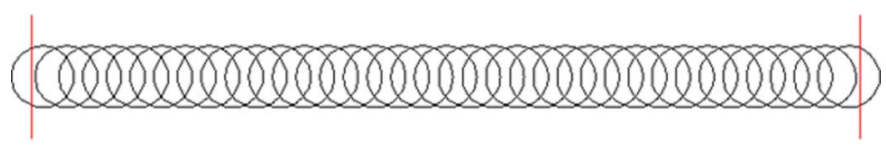

Fig. 2. Conventional arrangement of 35 sensors across a 1340-m wide river. Circles represent perimeter of sensor coverage; vertical lines denote river banks. Probability of detection $=0.869$.
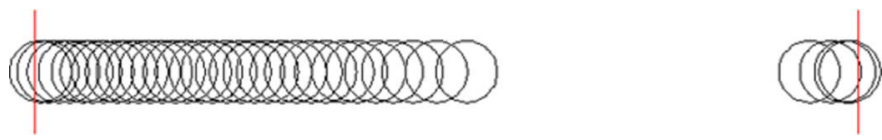

Fig. 3. Improved arrangement of 35 sensors, optimized using environmental modeling. Probability of detection $=0.971$.

of any particular arrangement of sensor placements as the total probability that a diver will be detected, should he attempt to cross the sensor line, found by integrating (3) across the river

$$
P\left(D^{T}\right)=\int_{\text {East_bank }}^{\text {West_bank }} P\left(D^{T}\right) p(x) \cdot d x .
$$

\section{RESULTS}

Using (5) as a fitness function, we can use a standard nonlinear optimization procedure to optimize the positions of a set of sensors. If 35 sensors are positioned in a conventional, equispaced linear pattern (Fig. 2), (4) yields a total probability of detection, $P\left(D^{T}\right)$ of 0.869 . In contrast, when we use our computational estuarine current model in conjunction with the diver preference curve, to optimize sensor placement (Fig. 3), we attain a significantly improved total probability of detection of 0.971 . Effectively, we have improved detection rates by removing sensors from regions of current that are impossible for a diver to navigate, and utilizing those sensors in more vulnerable parts of the river. Conversely, for a conventional sensor arrangement to achieve the same detection rate of $97 \%$ would require 58 sensors, so environmental optimization has achieved a $40 \%$ saving

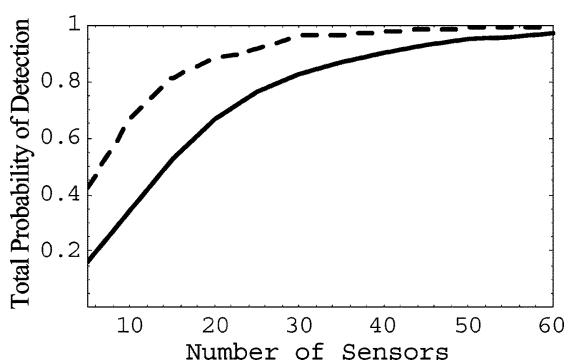

Fig. 4. Variation in total probability of detection with number of sensors. Top dashed line represents environmental optimization. Bottom solid line represents conventional equi-spaced arrangement.

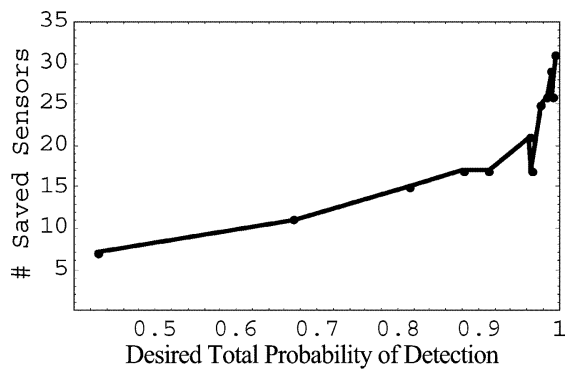

Fig. 5. Number of sensors saved using environmental optimization, while maintaining same detection rate as conventional arrangement, for various levels of desired detection rate.

in the number of required sensors. Fig. 4 shows how the benefits of environmental optimization vary with the total number of sensors. Fig. 5 shows the number of sensors which can be saved by utilizing the environmental optimization procedure, while maintaining the same detection rate as a conventional arrangement.

\section{FUTURE WORK}

Future work will extend this technique to detection in 2-D and 3-D spaces, examine control rules for mobile sensors in response to varying real-time current information, and may investigate the use of salinity forecast models for optimizing acoustic sensor placement.

\section{REFERENCES}

[1] D. Heeger, Signal Detection Theory. New York: New York Univ., 2003 [Online]. Available: http://www.cns.nyu.edu/ david/sdt/sdt.html

[2] S. A. Musman, P. E. Lehner, and C. Elsaesser, "Sensor planning for elusive targets," J. Math. Comput. Model., vol. 25, no. 3, pp. 103-115, 1997.

[3] The New York Harbor Observing and Prediction System (NYHOPS) [Online]. Available: http://onr.dl.stevens-tech.edu/webnyhos3/ 2003

[4] R. Stolkin and J. V. Nickerson, "Combining multiple autonomous mobile sensor behaviors using local clustering," presented at the MILCOM, Oct. 2005.

[5] S. Olariu and J. V. Nickerson, "Protecting with sensor networks: perimeters and axes," presented at the MILCOM, Oct. 2005.

[6] R. Stolkin, A. Sutin, S. Radhakrishnan, M. Bruno, B. Fullerton, A. Ekimov, and M. Raftery, "Feature based passive acoustic detection of a diver," presented at the SPIE Defense and Security Symp., 2006. 